\title{
OBSERVATIONAL LIMITS ON TYPE 1 ACTIVE GALACTIC NUCLEUS ACCRETION RATE IN COSMOS*
}

\author{
Jonathan R. Trump ${ }^{1}$, Chris D. Impey ${ }^{1}$, Brandon C. Kelly ${ }^{2,4}$, Martin Elvis ${ }^{2}$, Andrea Merloni ${ }^{3}$, Angela Bongiorno ${ }^{3,4}$, \\ Jared Gabor $^{1}$, Heng HaO ${ }^{2}$, Patrick J. McCarthy ${ }^{5}$, John P. Huchra ${ }^{2}$, Marcella Brusa $^{3}$, Nico Cappelluti ${ }^{3}$, \\ Anton Koekemoer ${ }^{6}$, Tohru Nagao ${ }^{7}$, Mara Salvato $^{8}$, and Nick Z. Scoville ${ }^{8}$ \\ ${ }^{1}$ Steward Observatory, University of Arizona, 933 North Cherry Avenue, Tucson, AZ 85721, USA \\ 2 Harvard-Smithsonian Center for Astrophysics, 60 Garden Street, Cambridge, MA 02138, USA \\ 3 Max Planck-Institut für Extraterrestrische Physik, Giessenbachstrasse 1, D-85748 Garching, Germany \\ ${ }^{4}$ University of Maryland, Baltimore County, 1000 Hilltop Circle, Baltimore, MD 21250, USA \\ 5 Observatories of the Carnegie Institute of Washington, Santa Barbara Street, Pasadena, CA 91101, USA \\ 6 Space Telescope Science Institute, 3700 San Martin Drive, Baltimore, MD 21218, USA \\ 7 Ehime University, 2-5 Bunkyo-cho, Matsuyama 790-8577, Japan \\ ${ }^{8}$ California Institute of Technology, MC 105-24, 1200 East California Boulevard, Pasadena, CA 91125, USA \\ Received 2009 February 17; accepted 2009 May 7; published 2009 June 30
}

\begin{abstract}
We present black hole masses and accretion rates for 182 Type 1 active galactic nuclei (AGNs) in COSMOS. We estimate masses using the scaling relations for the broad $\mathrm{H} \beta, \mathrm{Mg}$ II, and $\mathrm{C}$ IV emission lines in the redshift ranges $0.16<z<0.88,1<z<2.4$, and $2.7<z<4$.9. We estimate the accretion rate using an Eddington ratio $L_{I} / L_{\text {Edd }}$ estimated from optical and X-ray data. We find that very few Type 1 AGNs accrete below $L_{I} / L_{\text {Edd }} \sim 0.01$, despite simulations of synthetic spectra which show that the survey is sensitive to such Type 1 AGNs. At lower accretion rates the broad-line region may become obscured, diluted, or nonexistent. We find evidence that Type 1 AGNs at higher accretion rates have higher optical luminosities, as more of their emission comes from the cool (optical) accretion disk with respect to shorter wavelengths. We measure a larger range in accretion rate than previous works, suggesting that COSMOS is more efficient at finding low accretion rate Type 1 AGNs. However, the measured range in accretion rate is still comparable to the intrinsic scatter from the scaling relations, suggesting that Type 1 AGNs accrete at a narrow range of Eddington ratio, with $L_{I} / L_{\text {Edd }} \sim 0.1$.
\end{abstract}

Key words: galaxies: active - quasars: emission lines - quasars: general

Online-only material: color figures

\section{INTRODUCTION}

Supermassive black holes (SMBHs) reside in almost all local galaxies (Kormendy \& Richstone 1995; Richstone et al. 1998). The mass of the SMBH is observed to be tightly correlated with the mass, luminosity, and velocity dispersion of the host galaxy bulge (e.g., Magorrian et al. 1998; Gebhardt et al. 2000; Ferrarese \& Merritt 2000). SMBHs grow by accretion as active galactic nuclei (AGNs), and all massive galaxies have one or more of these active phases (Soltan 1982; Magorrian et al. 1998; Marconi et al. 2004). More luminous AGNs are observed to peak at higher redshift (Ueda et al. 2003; Brandt \& Hasinger 2005; Bongiorno et al. 2007), exhibiting "downsizing" by analogy to the preference of luminous and massive galaxies to form at high redshifts. Both downsizing and the correlations between SMBHs and the host bulge suggest that the growth of AGN and the formation of galaxies are directly connected through feedback (Silk \& Rees 1998; Di Matteo et al. 2005).

Understanding the role of AGNs in galaxy evolution requires measurements of SMBH mass and accretion over the cosmic time. The SMBH mass can be directly estimated by modeling the dynamics of nearby gas or stars, but this requires high spatial resolution and is limited to Hubble Space Telescope (HST) observations of nearby galaxies. Reverberation mapping of Type 1 AGN (with broad emission lines) uses the time lag between variability in the continuum and the broad-line region (BLR)

\footnotetext{
* Based on observations with the NASA/ESA Hubble Space Telescope, obtained at the Space Telescope Science Institute, which is operated by AURA Inc, under NASA contract NAS 5-26555; and based on data collected at the Magellan Telescope, which is operated by the Carnegie Observatories.

9 Hubble Fellow.
}

to estimate the radius of the BLR, $R_{\mathrm{BLR}}=c t_{\text {lag }}$ (for a review, see Peterson \& Bentz 2006). Then, if the BLR virially orbits the source of the continuum emission, the SMBH mass is $M_{\mathrm{BH}}=f R_{\mathrm{BLR}} v_{\mathrm{fwhm}}^{2}$, where $f$ represents the unknown BLR geometry and $v_{\mathrm{fwhm}}$ is the velocity width of the broad emission line. This technique has many potential systematic errors (Krolik 2001; Marconi et al. 2008), but its mass estimates agree with those from dynamical estimators (Davies et al. 2006; Onken et al. 2007) and those from the $M_{\mathrm{BH}}-\sigma *$ correlation (Onken et al. 2004; Greene \& Ho 2006). In principle, reverberation mapping can be applied to AGN at any redshift, but in practice, the need for many periodic observations has limited reverberation mapping mass estimates to only $\sim 35$ local AGNs.

Instead, the vast majority of AGNs mass estimates have come from a set of scaling relations. Reverberation mapping data led to the discovery that $R_{\mathrm{BLR}}$ correlates with the continuum luminosity (Kaspi et al. 2000), with $R_{\mathrm{BLR}} \sim L^{\alpha}$, where $\alpha \sim 0.5$ (Bentz et al. 2006; Kaspi et al. 2007). This allows for estimates of $M_{\mathrm{BH}}$ from single epoch spectra with scaling relations

$$
\log \left(\frac{M_{\mathrm{BH}}}{M_{\odot}}\right)=A+B \log \left(\lambda L_{\lambda}\right)+2 \log \left(v_{\mathrm{FWHM}}\right) .
$$

Some authors replace the continuum luminosity with the recombination line luminosity (Wu et al. 2004) or the FWHM with the second moment $\sigma$ (Collin et al. 2006), yielding minor systematic differences in estimated $M_{\mathrm{BH}}$ (Shen et al. 2008). While these scaling relations are based upon reverberation mapping of only local AGNs, the method is based upon the 
ability of the central engine to ionize the BLR (Kaspi et al. 2000), and there is no physical reason to suggest that the ionization of AGN should evolve with redshift (Dietrich \& Hamann 2004; Vestergaard 2004). Thus the scaling relations can be used to study the distribution and evolution of Type 1 AGN masses. Kollmeier et al. (2006) showed that Type 1 AGNs tend to accrete at a narrow range of Eddington ratio, typically $0.01 L_{\text {Edd }}<L<L_{\text {Edd }}$. Kollmeier et al. (2006) suggest a minimum accretion rate for Type 1 AGN, with AGNs of lower accretion rate observed as "naked" Type 2 AGNs without a BLR (Hopkins et al. 2009). Gavignaud et al. (2008) additionally suggest that lower luminosity Type 1 AGNs accrete less efficiently than brighter quasars.

In this work, we report black hole masses and study the demographics of 182 Type 1 AGNs in the Cosmic Evolution Survey (COSMOS; Scoville et al. 2007). We introduce the data and outline our spectral fitting in Section 2, and discuss the black hole masses, their associated errors, and our completeness in Section 3. We close with discussion of Type 1 AGN accretion rates in Section 4. All luminosities are calculated using $h=$ $0.70, \Omega_{M}=0.3, \Omega_{\Lambda}=0.7$.

\section{OBSERVATIONAL DATA}

\subsection{Sample}

COSMOS (Scoville et al. 2007) is a $2 \mathrm{deg}^{2}$ HST/ACS survey (Koekemoer et al. 2007) with ancillary deep multiwavelength observations. The depth of COSMOS over such a large area is particularly suited to the study of low-density, rare targets like AGNs. The most efficient way to select AGNs is by their $\mathrm{X}$-ray emission, and XMM-Newton observations of COSMOS (Cappelluti et al. 2009) reach fluxes of $1 \times 10^{-15} \mathrm{cgs}$ and $6 \times 10^{-15} \mathrm{cgs}$ in the $0.5-2 \mathrm{keV}$ and $2-10 \mathrm{keV}$ bands, respectively. The matching of X-ray point sources to optical counterparts is described by Brusa et al. (2007; M. Brusa et al. 2009, in preparation). Trump et al. (2009) performed a spectroscopic survey of XMM-selected AGNs in COSMOS, revealing 288 Type 1 AGNs with X-ray emission and broad emission lines in their spectra. Here, we investigate a chief physical property, the black hole mass, for the Type 1 AGNs in this survey.

From the Trump et al. (2009) sample, we choose the 182 Type 1 AGNs with high-confidence redshifts and with $\mathrm{H} \beta$, $\mathrm{Mg}$ II, or C IV present in the observed wavelength range. That is, we select only $z_{\text {conf }} \geqslant 3$ AGNs, empirically determined by Trump et al. (2009) to be at least $90 \%$ likely to have the correct classification and redshift. All of the Type 1 AGN spectra are dominated by blue power-law continua, with no obvious (beyond the noise) absorption line signature from the host galaxy. The broad emission line requirement restricts us to the redshift ranges $0.16<z<0.88,1<z<2.4$, and $2.7<z<4$.9. At these redshifts the AGN spectroscopy is $>90 \%$ complete to $i_{\mathrm{AB}}^{+}<22$ (see Figure 13 of Trump et al. 2009), where $i_{\mathrm{AB}}^{+}$is the $\mathrm{AB}$ magnitude from the COSMOS Canada-France-Hawaii Telescope (CFHT) observations. Our ability to measure a broad line width, however, is a slightly more complicated function of the spectral signal to noise. We characterize our completeness as a function of broad line width and signal-to-noise ratio $(\mathrm{S} / \mathrm{N})$ in Section 3.2.

The majority (133) of the 182 AGNs have spectra from Magellan/IMACS (Bigelow et al. 1998), with wavelength coverage from $5600-9200 \AA$ and $\sim 10 \AA$ resolution. The remaining bright 49 quasars have publicly available spectra from the Sloan Digital Sky Survey (SDSS) quasar catalog (Schneider et al. 2007), with 3800-9200 ̊ wavelength coverage and $\sim 3 \AA$ resolution. The resolution of both surveys is more than sufficient for measuring broad emission line widths; our narrowest broad emission line is $1300 \mathrm{~km} \mathrm{~s}^{-1}$ wide, compared to the resolution limits of IMACS $\left(\sim 600 \mathrm{~km} \mathrm{~s}^{-1}\right)$ and the SDSS $\left(\sim 200 \mathrm{~km} \mathrm{~s}^{-1}\right)$.

\subsection{Spectral Fitting}

The optical/UV spectrum of a Type 1 AGN can be roughly characterized as a power-law continuum, $f_{v} \propto v^{-\alpha}$, with additional widespread broad iron emission and broad emission lines (Vanden Berk et al. 2001). We followed Kelly et al. (2008) to model the spectra, using an optical Fe template from Veron-Cetty et al. (2004) and a UV Fe template from Vestergaard \& Wilkes (2001). Each spectrum was simultaneously fit with a power-law continuum and an iron template using the Levenberg-Marquardt method for nonlinear $\chi^{2}$ minimization. We calculated the continuum luminosity from the power-law fit parameters. Light from the host galaxy can artificially inflate the estimated AGN continuum luminosity, as shown by Bentz et al. (2009) for the 35 AGNs with reverberation mapping data. In particular, Bentz et al. (2009) find that at $5100 \AA$, host galaxies contribute $\sim 20 \%$ of the measured flux for AGNs with $\lambda L_{5100}>10^{44} \mathrm{erg} \mathrm{s}^{-1}$, and $\sim 45 \%$ of the flux for AGN of $\lambda L_{5100}<10^{44} \mathrm{erg} \mathrm{s}^{-1}$. We note that the expected host contamination at $L_{3000}$ or $L_{1350}$ is much smaller, since most host galaxies (excepting very active star-forming hosts) have much less flux blueward of the $4000 \AA$ A break. So for the majority of our sample, the 150 AGNs with masses measured from Mg II or C IV, we do not expect host contamination to be significant. However, the 15 AGNs with $\lambda L_{3000}<10^{44} \mathrm{erg} \mathrm{s}^{-1}$ and $\mathrm{H} \beta$-derived masses may have luminosity estimates overestimated by a factor of 2 , leading to black hole masses and Eddington ratios systematically overestimated by $\sim 0.15$ dex. Future work in COSMOS will use host decompositions from HST/ACS images of $z<1$ AGN to better characterize host contamination, but in this work we make no corrections for host galaxy flux.

To fit the broad emission lines we subtracted the continuum and $\mathrm{Fe}$ emission fits. Narrow absorption lines and narrow emission lines near the broad line (e.g., [O III] $\lambda 4959$ and $[\mathrm{O}$ III] $\lambda 5007$ near $\mathrm{H} \beta$ ) were fit by the sum of $1-2$ Gaussian functions and removed. Again following Kelly et al. (2008), each remaining broad emission line profile was fit by the sum of 1-3 Gaussian functions, minimizing the Bayesian information criterion (BIC, Schwartz 1979). Roughly, 2 Gaussians provided the best fit for $\sim 40 \%$ of line profiles, while 1 or 3 Gaussians each provided the best fit for $\sim 30 \%$ of line profiles. All fitting was interactive and inspected visually, and if the multipleGaussian fit revealed a narrow $\left(<600 \mathrm{~km} \mathrm{~s}^{-1}\right)$ line in an $\mathrm{H} \beta$ emission line, the component was attributed to non-BLR origins and was removed. The FWHM was calculated directly from the multiple-Gaussian fit in order to minimize the effects of noise in the original spectra. The multiple-Gaussian fit was robust to a variety of line profiles, and simulated spectra (see Section 3.2) revealed measured FWHM errors of only $\sigma_{\text {FWHM }} /$ FWHM $\sim 10 \%$.

Three examples of spectra with fitted continua and continuum-subtracted line profiles are shown in Figure 1. The spectra are representative of typical fits for each of the C IV, $\mathrm{Mg}$ II, and $\mathrm{H} \beta$ emission lines. At left the power law and iron emission fits are shown by the dashed blue lines. The right panel shows the multiple-Gaussian line profile fits as dashed blue lines, with the continuum-subtracted line profile shifted above by an arbitrary amount for clarity. The fit to the $\mathrm{H} \beta$ line profile in 

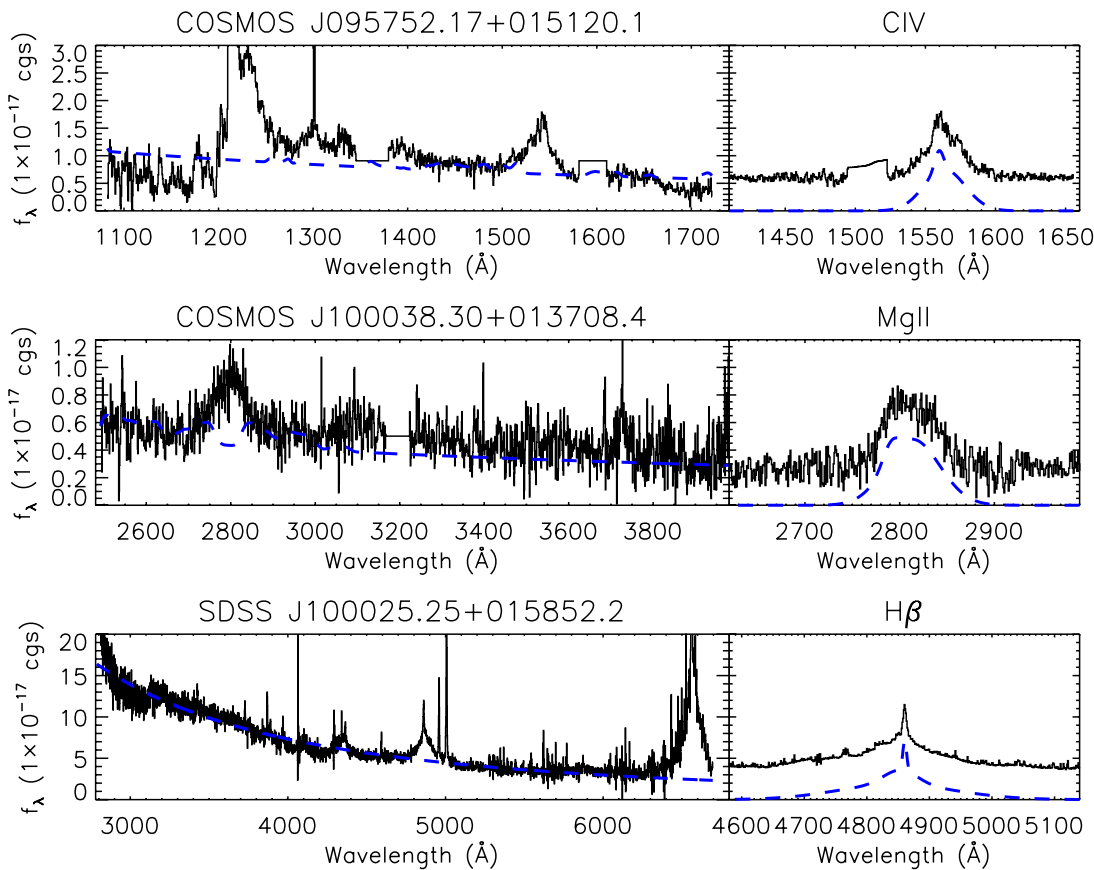

Figure 1. Three spectra, representing fits and line profiles for Type 1 AGN with C IV (top), Mg II (middle), and $\mathrm{H} \beta$ (bottom). In the left panels, the spectra are shown by black histograms, and the dashed blue lines are the power-law plus iron emission continuum fits. At right, the blue dashed line shows the multiple-Gaussian fit to the continuum-subtracted line profiles, which are shown in black and shifted above for clarity. The fit to the $\mathrm{H} \beta$ line profile in the lower right panel includes a narrow $\left(\sigma=433 \mathrm{~km} \mathrm{~s}^{-1}\right)$ Gaussian which was removed before calculating the broad emission line width. The top two spectra were observed with Magellan/IMACS and have $\sim 10 \AA$ resolution, while the bottom spectrum comes from the SDSS and has $\sim 3 \AA$ resolution.

(A color version of this figure is available in the online journal.)

Table 1

COSMOS Type 1 AGN Black Hole Mass Catalog

\begin{tabular}{|c|c|c|c|c|c|c|c|}
\hline $\begin{array}{c}\text { Object } \\
(\mathrm{J} 2000)\end{array}$ & Redshift & $\begin{array}{c}\mathrm{S} / \mathrm{N} \\
\left(\mathrm{pixel}^{-1}\right)^{\mathrm{a}}\end{array}$ & $\begin{array}{c}\log \left(\lambda L_{3000} \AA\right) \\
\left(\mathrm{erg} \mathrm{s}^{-1}\right) \\
\end{array}$ & $\begin{array}{c}\log \left(L_{0.5-2} \mathrm{keV}\right) \\
\left(\mathrm{erg} \mathrm{s}^{-1}\right)^{\mathrm{b}}\end{array}$ & Line & $\begin{array}{c}\text { FWHM } \\
\left(\mathrm{km} \mathrm{s}^{-1}\right)\end{array}$ & $\begin{array}{c}\log \left(M_{\mathrm{BH}}\right) \\
{\left[M_{\odot}\right]} \\
\end{array}$ \\
\hline SDSS J095728.34+022542.2 & 1.54 & 7.0 & 45.00 & 44.47 & Mg II & 4491 & 8.665 \\
\hline SDSS J095728.34+022542.2 & 1.54 & 7.0 & 45.00 & 44.47 & C IV & 2776 & 8.135 \\
\hline SDSS J095743.33+024823.8 & 1.36 & 3.4 & 44.60 & 43.50 & Mg II & 3472 & 8.243 \\
\hline COSMOS J095752.17+015120.1 & 4.17 & 7.3 & 45.19 & 44.36 & C IV & 4603 & 8.656 \\
\hline COSMOS J095753.49+024736.1 & 3.61 & 4.8 & 44.14 & 44.53 & C IV & 2629 & 7.997 \\
\hline SDSS J095754.70+023832.9 & 1.60 & 8.0 & 45.17 & 43.69 & $\mathrm{C}$ IV & 6384 & 8.946 \\
\hline SDSS J095755.08+024806.6 & 1.11 & 8.7 & 44.92 & 44.08 & Mg II & 3574 & 8.426 \\
\hline COSMOS J095755.34+022510.9 & 2.74 & 3.2 & 44.38 & -1.00 & C IV & 3879 & 8.074 \\
\hline COSMOS J095755.48+022401.1 & 3.10 & 19.9 & 45.28 & 45.00 & C IV & 3527 & 8.436 \\
\hline
\end{tabular}

Notes.

a The SDSS spectra have 3 pixels per resolution element, and the Magellan/IMACS spectra have 5 pixels per resolution element.

${ }^{\mathrm{b}}$ AGNs with no soft X-ray detection have an entry of -1.00 for $\log \left(L_{0.5-2 \mathrm{keV}}\right)$.

the bottom right panel includes a narrow $\left(\sigma=433 \mathrm{~km} \mathrm{~s}^{-1}\right)$ Gaussian which is not associated with the BLR and was removed. Even for noisy spectra like the middle panel, the spectral fitting provides a robust continuum and isolates the emission line.

\section{ESTIMATED BLACK HOLE MASSES}

We estimate black hole masses using our measured broad line velocity widths and the scaling relations of $\mathrm{M}$. Vestergaard et al. (2009, in preparation) for $\mathrm{Mg}$ II and Vestergaard \& Peterson (2006) for $\mathrm{H} \beta$ and $\mathrm{C}$ IV. These relations all take the form of Equation (1), with $\lambda L_{\lambda}$ in units of $10^{44} \mathrm{erg} \mathrm{s}^{-1}$ and $v_{\mathrm{FWHM}}$ in units of $1000 \mathrm{~km} \mathrm{~s}^{-1} ; A=6.91, B=0.50$, and $\lambda=5100 \AA$ for $\mathrm{H} \beta ; A=6.86, B=0.50$, and $\lambda=3000 \AA$ for $\mathrm{Mg} \mathrm{II}$; $A=6.66, B=0.53$, and $\lambda=1350 \AA$ for C IV. The Mg II relation was derived from SDSS quasars with both $\mathrm{C}$ IV and $\mathrm{Mg}$ II in the spectrum, and it is designed to produce black hole masses consistent with those measured from C IV. In our sample, we measure $\mathrm{H} \beta$ for 32 AGNs, Mg II for 134 AGNs, and C IV for 38 AGNs (19 SDSS AGNs have both Mg II and C IV, and 3 have both $\mathrm{H} \beta$ and $\mathrm{Mg}$ II). AGNs with estimates of $M_{\mathrm{BH}}$ from two different emission lines are treated as two separate objects in our subsequent analyses.

Table 1 presents the catalog of black hole masses and line measurements. AGNs with both $\mathrm{Mg}$ II and $\mathrm{C}$ IV or both $\mathrm{H} \beta$ 


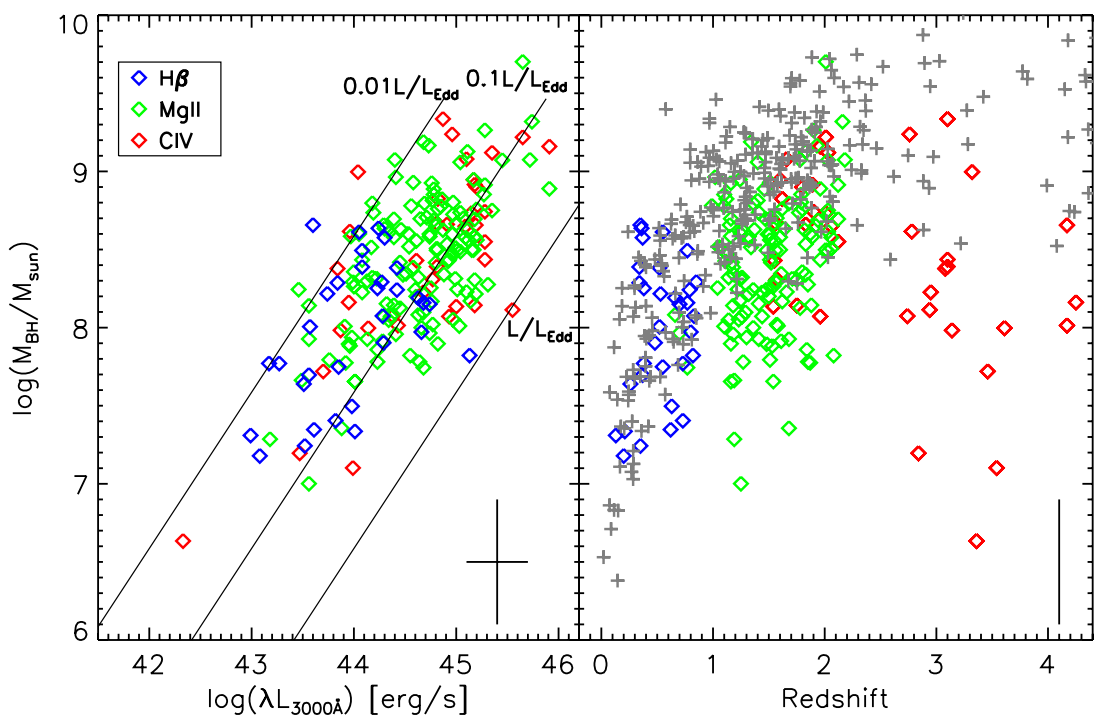

Figure 2. Black hole mass vs. optical luminosity and redshift for 182 Type 1 AGNs in COSMOS. The black hole masses are derived from the broad line velocity widths of $\mathrm{H} \beta$ (blue diamonds), Mg II (green diamonds), and C IV (red diamonds) depending on the redshift (Vestergaard \& Peterson 2006, M. Vestergaard et al. 2009, in preparation). The black hole masses have uncertainties of $\sim 0.4$ dex from the scaling relations (Krolik 2001; Shen et al. 2008), as shown by the typical error bars are shown in the lower right of each panel (the redshift error is $\leqslant 1 \%$ ). The diagonal tracks at left represent Eddington ratios, assuming a bolometric correction of $L_{\text {bol }}=5\left(\lambda L_{3000}\right)$ (Richards et al. 2006). At right the gray crosses show scaling relation masses of SDSS quasars (Kelly et al. 2008). The depth of COSMOS allows us to probe low mass and weakly accreting SMBHs, revealing that $L / L_{\text {Edd }} \lesssim 0.01$ Type 1 AGNs do not exist.

(A color version of this figure is available in the online journal.)
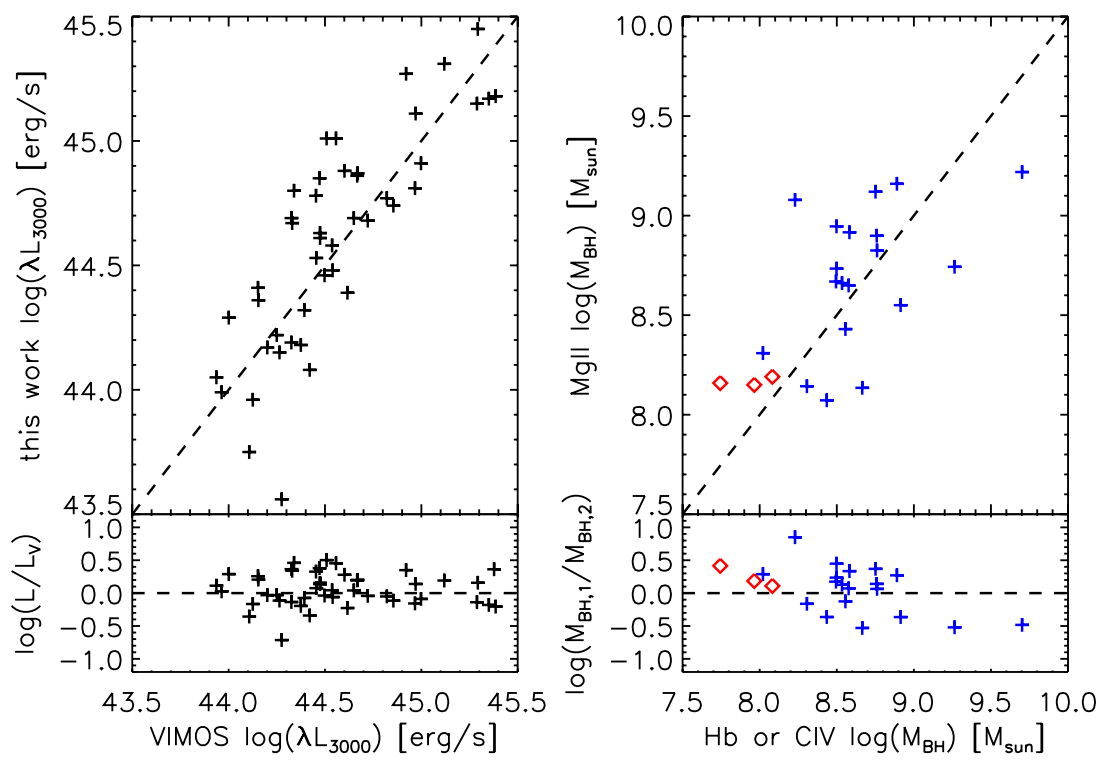

Figure 3. At left, the luminosity estimates for 48 AGNs in this work are compared to estimates of the same objects from A. Merloni (2009, in preparation), which have independent redshifts from VLT/VIMOS spectra and are calculated from a fit to the multiwavelength SED. The scatter between the two luminosity estimates is $\sigma=0.25 \mathrm{dex}$, which contributes very little to the overall scatter in $M_{\mathrm{BH}}$. At right, $M_{\mathrm{BH}}$ estimates are compared for the 22 spectra with two emission lines (either $\mathrm{Mg}$ II and $\mathrm{H} \beta$, red diamonds, or $\mathrm{Mg}$ II and $\mathrm{C}$ IV, blue crosses). The scatter between separate $M_{\mathrm{BH}}$ estimates is only $\sigma=0.36$ dex, indicative that the intrinsic scatter of $\sim 0.4$ dex dominates the $M_{\mathrm{BH}}$ error.

(A color version of this figure is available in the online journal.)

and $\mathrm{Mg}$ II present have two entries in Table 1, one for each emission line. The full catalog then contains 204 entries: 182 Type 1 AGNs with $M_{\mathrm{BH}}$ estimates, 22 of which have two sets of broad emission line measurements. The black hole masses are shown with continuum luminosity (calculated from the power-law fit) and redshift in Figure 2. The diagonal tracks in the figure represent Eddington ratios using a bolometric correction of 5 for $\lambda L_{3000}$ (Richards et al. 2006). We also show a comparison sample of brighter SDSS quasars (Kelly et al. 2008) in order to highlight the lower black hole masses probed by COSMOS.

\subsection{Error}

The scaling relations have uncertainties of $\sim 0.4 \mathrm{dex}$, although there may be larger systematic uncertainties (Krolik 2001; Collin et al. 2006; Fine et al. 2008; Marconi et al. 2008). Measurement errors in the luminosity and emission line FWHM also contribute, but the uncertainty from the scaling relations dominates. We test the luminosity error in the left panel of Figure 3 , which compares the luminosity estimates from this work to duplicate estimates from A. Merloni (2009, in preparation). The luminosity estimates of A. Merloni (2009, in preparation) 
use independent redshifts from VLT/VIMOS spectra and are calculated from a fit to the IR to X-ray multiwavelength spectral energy distribution, instead of from the optical spectrum itself (as in this work). The scatter between the two luminosity estimates is $\sigma=0.25 \mathrm{dex}$. The Type 1 AGNs in COSMOS have an average variability of $\sim 0.15 \mathrm{dex}$ (Salvato et al. 2009), the remaining luminosity scatter can be attributed to the different methods of estimates. Since $M_{\mathrm{BH}} \sim L^{0.5}$, our luminosity error contributes very little to the overall $M_{\mathrm{BH}}$ uncertainty.

Line measurements of synthetic spectra (described in Section 3.2 ) show that our FWHM error is only $\sigma_{\text {FWHM }} /$ FWHM $\sim 10 \%$ at $i_{\mathrm{AB}}^{+} \sim 22$. The right panel of Figure 3 compares the duplicate estimates of $M_{\mathrm{BH}}$ for spectra with two broad emission lines. Red diamonds indicate spectra with both $\mathrm{Mg}$ II and $\mathrm{H} \beta$, while blue crosses indicate both $\mathrm{Mg}$ II and C IV. The scatter between the different estimates is only $\sigma=0.36$ dex, nearly the same as the expected intrinsic scatter for $M_{\mathrm{BH}}$. This suggests that the statistical error in the mass estimators is not correlated to the choice of emission line (see also Kelly \& Bechtold 2007). If there were systematic offsets in the mass estimators, they would cause a constant shift in the mass estimate for each line, and therefore would not contribute to the scatter between two lines. The statistical intrinsic scatter, however, would not "cancel" in such a way. Because the scatter between lines is comparable to the expected intrinsic scatter, our estimates of $M_{\mathrm{BH}}$ probably do not have significant systematic errors.

\subsection{Completeness}

Previous works (Trump et al. 2009) tested the completeness of the Type 1 AGN sample, with simulated spectra showing that Type 1 AGNs are correctly identified (with high-confidence redshifts) at $90 \%$ completeness to $\mathrm{S} / \mathrm{N} \gtrsim 2.87$. But even for correctly identified Type 1 AGNs, our measurements of $M_{\mathrm{BH}}$ are roughly limited by spectral $\mathrm{S} / \mathrm{N}$ and FWHM, since we cannot identify or measure broad emission lines for spectra that have lines so broad that they become confused with noise or the Fe emission. To test these limits, we create 100 synthetic spectra of Type 1 AGNs with Mg II in the observed wavelength range. We choose $\mathrm{Mg}$ II because it is the most common line used for our $M_{\mathrm{BH}}$ calculations and also because it is the broad emission line most contaminated by widespread iron emission. These synthetic spectra are formed by making a composite of all $1<z<2.4$ observed spectra, then removing the rest-frame $2700 \AA<\lambda<2900 \AA \mathrm{Mg}$ II region. To each of the 100 spectra we then re-add a $\mathrm{Mg}$ II region with random FWHMs and line areas, and then each spectrum has random noise added. We choose the line areas and noise to be normally distributed in the ranges of measured line area and $\mathrm{S} / \mathrm{N}$ in the original spectra, while the FWHMs are chosen to probe our sensitivity to the broadest line widths, FWHM $\gtrsim 10,000 \mathrm{~km} \mathrm{~s}^{-1}$.

We show the FWHM and $\mathrm{S} / \mathrm{N}$ of both the observed and simulated spectra in Figure 4. The blue diamonds and red squares represent the simulated spectra that we successfully measure and those we miss, respectively. The vertical dotted line shows the sample's $90 \%$ completeness limit for correctly identifying high-confidence Type 1 AGNs. The dashed line shows the limit to where we can measure broad emission lines, corresponding to $>95 \%$ completeness since only one "missed" synthetic spectrum lies below the line. The observed Type 1 AGNs tail off well before the dashed line. Translating FWHM and magnitude into black hole mass, as an example, a Type 1 AGN with $i_{\mathrm{AB}}^{+} \sim 22$ at $z \sim 1.7$ with a $\mathrm{Mg}$ II profile of $\mathrm{FWHM}=10,000 \mathrm{~km} \mathrm{~s}^{-1}$ would have $\log \left(M_{\mathrm{BH}} / M_{\odot}\right) \sim 10.6$

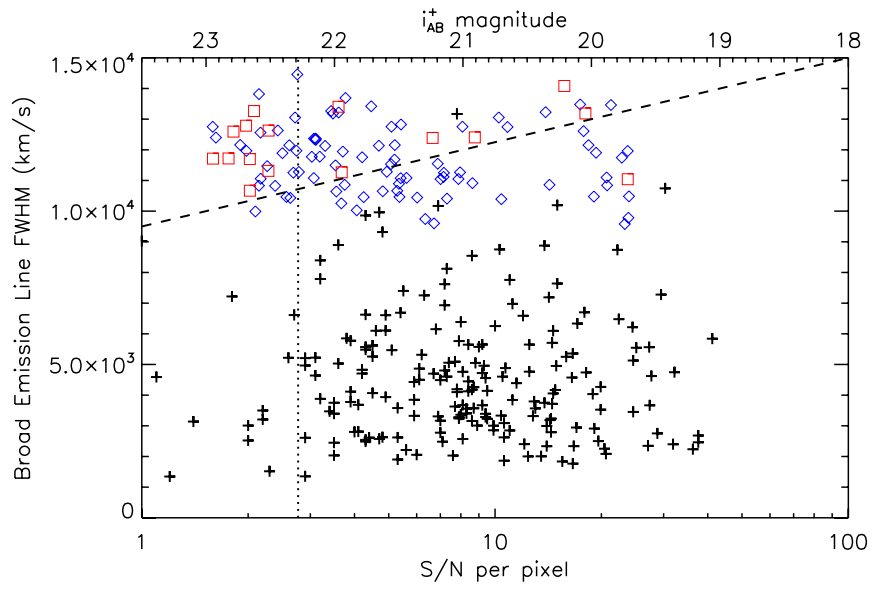

Figure 4. Broad emission line FWHM and $\mathrm{S} / \mathrm{N}$ for our 182 Type 1 AGNs are shown as black crosses. The upper axis shows the $i_{\mathrm{AB}}^{+}$magnitude scale which roughly corresponds to the $S / N$ (from Figure 11 of Trump et al. 2009). Also shown are the measured FWHM and S/N for 100 simulated Type 1 AGN spectra: blue diamonds represent spectra we can correctly identify and measure, while red squares represent synthetic spectra that we incorrectly identify or cannot properly measure FWHM. The dashed line shows the limit to which we correctly identify and measure $>95 \%$ of synthetic spectra. The dotted vertical line shows the $90 \%$ completeness to identifying and assigning high-confidence redshifts to Type 1 AGN, from additional simulations (Trump et al. 2009). The lack of high-FWHM AGN identified in COSMOS is not from selection effects, since we can correctly identify and measure synthetic Type 1 AGN with much broader lines at $\mathrm{S} / \mathrm{N}>3$.

(A color version of this figure is available in the online journal.)

and $L / L_{\text {Edd }} \sim 0.0002$. We do not detect such objects in our sample, yet our simulations show that they do not lie beyond our detection limits.

\section{DISCUSSION}

In Figure 2, all Type 1 AGNs lie within the region of $0.01 \lesssim L / L_{\text {Edd }} \lesssim 1$, a result supported by Kollmeier et al. (2006). This implies that the broad emission line region of Type 1 AGN might become undetectable as the accretion drops below $L \sim 0.01 L_{\text {Edd }}$. Such objects might be observed as unobscured Type 2 AGNs, the possible remnants of "dead" Type 1 AGN whose accretion disk geometries changed as their accretion rates fell (e.g., Hopkins et al. 2009). Or these low accretion rate AGNs may be diluted, with their emission falling below the light of their host galaxy. The AGN emission may also be unable to blow out local obscuring material, causing their BLR to lie undetected behind obscuration.

To study the accretion rates of our AGNs, we calculate the intrinsic luminosity from our measured $\lambda L_{3000}$ and $L_{0.5-2} \mathrm{keV}$, using the relations of Marconi et al. (2004),

$$
\begin{gathered}
\log \left[L_{I} /\left(\lambda L_{3000}\right)\right]=0.65-0.067 \mathcal{L}+0.017 \mathcal{L}^{2}-0.0023 \mathcal{L}^{3}, \\
\log \left(L_{I} / L_{0.5-2}\right)=1.65+0.22 \mathcal{L}+0.012 \mathcal{L}^{2}-0.0015 \mathcal{L}^{3} .
\end{gathered}
$$

Here $\mathcal{L}=\log \left(L_{I}\right)-45.58$ and all luminosities are in units of erg $\mathrm{s}^{-1}$. The intrinsic luminosity $L_{I}$ is designed to be a bolometric luminosity which excludes reprocessed (IR) emission, so that the Eddington ratio $L_{I} / L_{\text {Edd }}$ represents a robust measure of the accretion onto the black hole. We use the Newton method to solve each equation for $L_{I}$ from $\lambda L_{3000}$ and $L_{0.5-2}$. We then average the two values of $L_{I}$ for our final value (excepting 7 AGNs, where we estimate $L_{I}$ from $\lambda L_{3000}$ only because they lack soft X-ray detections). 


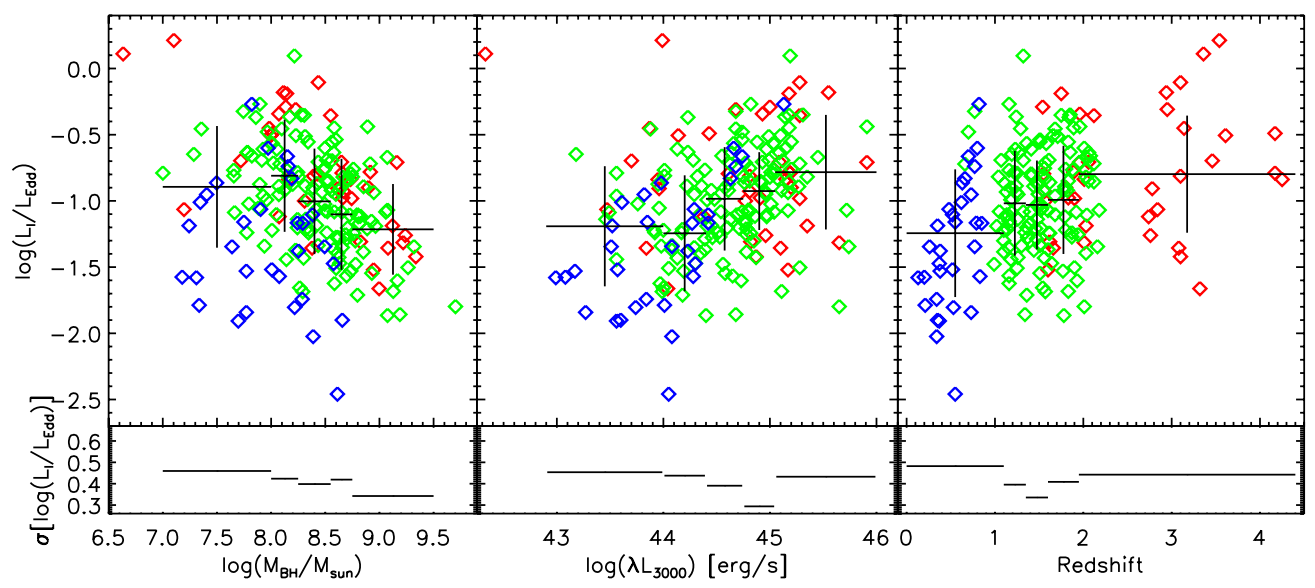

Figure 5. Eddington ratio (accretion rate) with black hole mass, luminosity, and redshift for our Type 1 AGN. Eddington ratio was calculated using an intrinsic luminosity $L_{I}$ estimated from $\lambda L_{3000}$ and $L_{0.5-2 \mathrm{keV}}$ using the relations of Marconi et al. (2004). Diamonds represent individual objects with masses estimated from $\mathrm{H} \beta$ (blue), $\mathrm{Mg}$ II (green), and $\mathrm{C}$ IV (red). The large crosses in the top plots show the mean accretion rate in each bin of $M_{\mathrm{BH}}$ or redshift, while the gray lines at the bottom show the standard deviation (the square root of the second moment) in each bin. The dispersion deviation is also shown by the vertical error bar in the top plots. Bins were chosen to each have the same number of objects. Selection effects cause the apparent trends of $L_{I} / L_{\mathrm{Edd}}$ decreasing with $M_{\mathrm{BH}}$ and increasing with redshift, but the increase of $L_{I} / L_{\text {Edd }}$ with $\lambda L_{3000}$ is a physical effect caused by changes in the accretion disk. The dispersion is generally $\sim 0.4$ dex, higher than that of previous, less sensitive surveys.

(A color version of this figure is available in the online journal.)

We show the Eddington ratios of our Type 1 AGNs with $M_{\mathrm{BH}}, \lambda L_{3000}$, and redshift in Figure 5. The diamonds show individual objects, while the solid lines show the means and scatter in equal-sized bins. The scatter (the standard deviation of the mean) is calculated as the square root of the second moment of the data in each bin. Our mean Eddington ratio for all Type 1 AGNs is $L_{I} / L_{\mathrm{Edd}} \sim 0.1$, lower than the value of $L_{I} / L_{\mathrm{Edd}} \sim 0.3$ found in previous surveys (Kollmeier et al. 2006; Gavignaud et al. 2008). This is partly explained by the depth of COSMOS: Kollmeier et al. (2006) noted that their $R \leqslant 21.5$ AGES sample was only complete to $L_{I} / L_{\mathrm{Edd}} \sim 0.1$, while our simulations in Section 3.2 show that COSMOS can reach much weaker accretors. In addition, the center panel of Figure 5 shows that accretion rate may increase with optical/ UV luminosity, suggesting an additional reason for our lower mean Eddington ratio: most of the Kollmeier et al. (2006) and Gavignaud et al. (2008) AGNs have $\lambda L_{3000}>10^{45} \mathrm{erg} \mathrm{s}^{-1}$, where our AGNs have $L_{I} / L_{\mathrm{Edd}} \sim 0.2$. The majority of our AGNs have $\lambda L_{3000}<10^{45} \mathrm{erg} \mathrm{s}^{-1}$ and so we find a lower mean accretion rate.

The apparent decrease in accretion rate with black hole mass and the apparent increase in accretion rate with redshift can be explained by selection effects: low accretion rate AGNs are more difficult to detect if they are also low mass or at higher redshift. The increase in accretion rate with optical luminosity, however, is also observed by Gavignaud et al. (2008) and is probably a physical effect. We performed a linear regression analysis of the correlation between accretion rate and optical luminosity using the publicly available IDL program linmix_err.pro (Kelly 2007). Using errors of 0.25 dex in $\log \left(\lambda L_{3000}\right)$ and $0.4 \mathrm{dex}$ in $\log \left(L_{I} / L_{\mathrm{Edd}}\right)$, linear regression indicates that $\log \left(L_{I} / L_{\text {Edd }}\right) \sim(0.28 \pm 0.06) \log \left(\lambda L_{3000}\right)$. In other words, accretion rate is correlated with optical luminosity at the $4.8 \sigma$ level. As a Type 1 AGN increases in accretion rate, its optical emission becomes a larger fraction of its total bolometric output because its cool accretion disk emits more brightly. This is consistent with the results of Kelly et al. (2008), which show that $\alpha_{O X}$ (the ratio between optical/UV and X-ray flux) becomes more X-ray quiet with accretion rate. Thus, a more rapidly accreting Type 1 AGN has more of its emission in its cool (optical) disk than in its hot (X-ray) corona, possibly because the disk grows larger or thicker as the accretion rate approaches the Eddington limit.

The scatter (square root of the second moment) in $L_{I} / L_{\mathrm{Edd}}$, as shown in the bottom panels of Figure 5, is typically only $\sim 0.4$ dex in each bin. This is greater than previously measured dispersions (Kollmeier et al. 2006; Gavignaud et al. 2008; Fine et al. 2008), and indicates that COSMOS is more sensitive to low accretion rate Type 1 AGNs than previous studies. Yet it is remarkable that the dispersion is not larger than the scatter from the scaling relations: the intrinsic dispersion in Eddington ratio might then be $\sim 0$, with nearly all Type 1 AGNs of a given mass, luminosity, and/or redshift accreting at a very narrow range of of accretion rates. Fine et al. (2008) note that at such low measured dispersions, if the intrinsic dispersion in accretion rate is much greater than 0 , then the BLR cannot be in a simple virial orbit. Accurate scaling relations would then require a luminosity-dependent ionization parameter (Marconi et al. 2008) or a more complex BLR geometry (Fine et al. 2008). We note, however, that the myriad uncertainties involved in estimating $L_{I} / L_{\text {Edd }}$ make concrete conclusions difficult. And although we find significant evidence that $L_{I} / L_{\text {Edd }}<0.01$ Type 1 AGNs do not exist (or are very rare), the spectroscopic flux limit may still miss some $L_{I} / L_{\mathrm{Edd}} \sim 0.01 \mathrm{AGNs}$ at lower luminosities.

\section{SUMMARY}

The black hole masses of Type 1 AGNs in COSMOS indicate that Type 1 AGNs accrete at a narrow range of high efficiencies, $L_{I} / L_{\mathrm{Edd}} \sim 0.1$. When the accretion rate of an AGN lowers, less of its luminosity is emitted optically. When a Type 1 AGN accretion rate drops below $L_{I} / L_{\text {Edd }} \sim 0.01$, the BLR becomes invisible, due to obscuration, dilution, or an altered accretion disk geometry. We additionally measure higher dispersions in accretion rate than previous, less sensitive surveys, although the dispersion is still no larger than the intrinsic uncertainty in the scaling relations. Kelly et al. (2008) find that the bolometric 
correction depends on black hole mass, and Vasudevan \& Fabian (2009) find that it correlates with Eddington ratio. This makes characterizing the distributions of $L_{I} / L_{\text {Edd }}$ and its scatter rather difficult. We partially mitigate the systematic uncertainties by using both $\lambda L_{3000}$ and $L_{0.5-2}$ to estimate $L_{I}$. Future work in COSMOS will use more accurate bolometric luminosities calculated from the full multiwavelength data set.

We thank Brad Peterson for helpful discussions on emission line measurements and Misty Bentz for comments on host contamination. We thank Marianne Vestergaard for providing the Fe templates and for discussions on the scaling relations. Amy Stutz and Aleks Diamond-Stanic provided useful discussions on statistics. J.R.T acknowledges support from NSF ADP grant NNX08AJ28G and an ARCS fellowship. B.C.K acknowledges support from NASA through Hubble Fellowship grant \#HF-01220.01 awarded by the Space Telescope Science Institute, which is operated by the Association of Universities for Research in Astronomy, Inc., for NASA, under contract NAS 5-26555.

\section{REFERENCES}

Bentz, M. C., Peterson, B. M., Pogge, R. W., \& Vestergaard, M. 2009, ApJ, 694, L166

Bentz, M. C., Peterson, B. M., Pogge, R. W., Vestergaard, M., \& Onken, C. A. 2006, ApJ, 644, 133

Bigelow, B. C., Dressler, A. M., Shectman, S. A., \& Epps, H. W. 1998, in Proc. SPIE 3355, Optical Astronomical Instrumentation, ed. S. D’Odorico (Bellingham, WA: SPIE), 225

Bongiorno, A., et al. 2007, A\&A, 472, 443

Brandt, W. N., \& Hasinger, G. 2005, ARA\&A, 43, 827

Brusa, M., et al. 2007, ApJS, 172, 353

Cappelluti, N., et al. 2009, A\&A, 497, 635

Collin, S., Kawaguchi, T., Peterson, B. M., \& Vestergaard, M. 2006, A\&A, 456, 75

Davies, R. I., et al. 2006, ApJ, 646, 754

Dietrich, M., \& Hamann, F. 2004, ApJ, 611, 761

Di Matteo, T., Springel, V., \& Hernquist, L. 2005, Nature, 433, 604

Ferrarese, L., \& Merritt, D. 2000, ApJ, 593, L9
Fine, S., et al. 2008, MNRAS, 390, 1413

Gavignaud, I., et al. 2008, A\&A, 492, 637

Gebhardt, K., et al. 2000, ApJ, 539, 13

Greene, J. E., \& Ho, L. C. 2006, ApJ, 641, 21

Hopkins, P. F., Hickox, R., Quataert, E., \& Hernquist, L. 2009, MNRAS submitted (arXiv:0901.2936)

Kaspi, S., Brandt, W. N., Maoz, D., Netzer, H., Schneider, D. P., \& Shemmer O. 2007, ApJ, 659, 997

Kaspi, S., Smith, P. S., Netzer, H., Maoz, D., Jannuzi, B. T., \& Giveon, U. 2000, ApJ, 533, 631

Kelly, B. C. 2007, ApJ, 665, 1489

Kelly, B. C., \& Bechtold, J. 2007, ApJS, 168,

Kelly, B. C., Bechtold, J., Trump, J. R., Vestergaard, M., \& Siemiginowska, A. 2008, ApJS, 176, 355

Koekemoer, A. M., et al. 2007, ApJS, 172, 196

Kollmeier, J. A., et al. 2006, ApJ, 648, 128

Kormendy, J., \& Richstone, D. 1995, ARA\&A, 33, 581

Krolik, J. H. 2001, ApJ, 551, 72

Magorrian, J., et al. 1998, AJ, 115, 2285

Marconi, A., Risaliti, G., Gilli, R., Hunt, L. K., Maiolino, R., \& Salvati, M. 2004, MNRAS, 351, 169

Marconi, A., et al. 2008, ApJ, 678, 693

Onken, C. A., Ferrarese, L., Merritt, D., Peterson, B. M., Pogge, R. W., Vestergaard, M., \& Wandel, A. 2004, ApJ, 615, 645

Onken, C. A., et al. 2007, ApJ, 670, 105

Peterson, B. M., \& Bentz, M. C. 2006, New Astron. Rev., 50, 796

Richards, G. T., et al. 2006, ApJ, 166, 470

Richstone, D., et al. 1998, Nature, 395, 14

Salvato, M., et al. 2009, ApJ, 690, 1250

Schneider, D. P., et al. 2007, AJ, 130, 367

Schwartz, G. 1979, Ann. Stat., 6, 461

Scoville, N., et al. 2007, ApJS, 172, 38

Shen, Y., Greene, J. E., Strauss, M. A., Richards, G. T., \& Schneider, D. P. 2008, ApJ, 680, 169

Silk, J., \& Rees, M. J. 1998, A\&A, 331, 1

Soltan, A. 1982, MNRAS, 200, 115

Trump, J. R., et al. 2009, ApJ, 696, 1195

Ueda, Y., et al. 2003, ApJ, 598, 886

Vanden Berk, D. E., et al. 2001, AJ, 122, 549

Vasudevan, R. V., \& Fabian, A. C. 2009, MNRAS, 392, 1124

Veron-Cetty, M.-P., Joly, M., \& Veron, P. 2004, A\&A, 417, 515

Vestergaard, M. 2004, ApJ, 601, 676

Vestergaard, M., \& Peterson, B. M. 2006, ApJ, 641, 689

Vestergaard, M., \& Wilkes, B. J. 2001, ApJS, 134, 1

Wu, X.-B., Wang, R., Kong, M. Z., Liu, F. K., \& Han, J. L. 2004, A\&A, 424, 793 